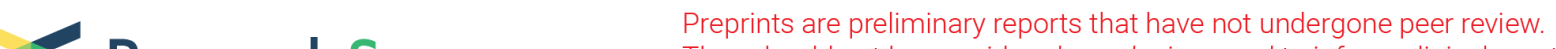 Research Square They should not be considered conclusive, used to inform clinical practice, or referenced by the media as validated information.
}

\section{Baduanjin Exercise May Improve the Anxiety and Insomnia in COVID-2019 Patients: A Case-Control Study}

\section{Ming-Gui Chen}

the second clinica college of guangzhou university of chinese medicine

\section{Yinlong Qiu}

the second clinical college of guangzhou uniersity of chinese medicine

\section{Haizhen Chen}

the second clinical college of guangzhou university of chinese medicine

\section{Erhui Chen}

the second clinical college of guangzhou university of chinesemedicine

\section{Rui-Xiang Zeng}

the second clinical college of guangzhou university of chinese medicine

\section{Qiaomei Wu}

the second clinical college of guangzhou university of chinese medicine

\section{Xiaoxuan Zhang}

the second clinical college of guangzhou university of chinese medicine

\section{Min-Zhou Zhang}

the second clinical college of guangzhou university of chinese medicine

MeiZhen Lin ( $\nabla$ szylmz@163.com)

The second clinical college of guangzhou university of chinese medicine https://orcid.org/0000-00032317-3337

\section{Research}

Keywords: Baduanjin, anxiety, insomnia, COVID-2019 patients

Posted Date: July 20th, 2020

DOl: https://doi.org/10.21203/rs.3.rs-42755/v1

License: (c) (1) This work is licensed under a Creative Commons Attribution 4.0 International License.

Read Full License 


\section{Abstract}

Background: Since December 2019, an epidemic caused by novel coronavirus

(2019-nCoV) infection has occurred unexpectedly in China. Because of the sudden nature of the outbreak and the infectious power of the virus, it will inevitably cause people anxiety and other stress reactions. Previous studies showed that Baduanjin exercise was effective for people in anxiety and insomnia. The purpose of this study was to evaluate the potential benefits of Baduanjin exercise on the anxiety and insomnia in COVID-2019 patients.

Methods: This is a Case-Control Study. The COVID-2019 patients including 39 Baduanjin exercises or willing to do Baduanjin exercises and 39 age-and gender-matched nonexercising controls. The anxiety and insomnia in COVID-2019 patients were measured by using the GAD-7 and SMH Sleep Questionnaire at baseline and discharge.

Results: In the study, the Paired T-test showed that two groups had improved the GAD-7 scores and SMH Sleep Questionnaire compared with baseline at discharge. Baseline results showed there were no significant differences in the GAD-7 scores and SMH Sleep Questionnaire between the two groups. However, the significant differences found in the Baduanjin group included a $43.9 \%$ lower $(p<0.001)$ in the GAD-7 score and an approximately $75.9 \%$ higher $(p=0.003)$ in SMH Sleep Questionnaire score compare with the control group at discharge.

Conclusion: The Baduanjin exercise may improve the anxiety and insomnia in COVID-2019 patients. It can also be used as a form of rehabilitation exercise for discharged patients or patients isolated at home.

Trial Registration: ChiCTR2000030528.

\section{Background}

The novel coronavirus 2019 currently designated as COVID-19 is in the limelight since the beginning of New Year 2020 [1]. The World Health Organization has declared that the outbreak of COVID-19 is a worldwide pandemic, raising concerns of widespread panic and increasing anxiety in individuals subjected to the real or perceived threat of the virus [2]. Owing to the lack of immunity to this new strain of coronavirus, a large number of people at large are susceptible to it. The clinical features of COVID-19 are varied, ranging from an asymptomatic state to acute respiratory distress syndrome and multi-organ dysfunction. Common symptoms at the onset of illness were fever, cough, and myalgia or fatigue. Less common symptoms were sputum production, headache, hemoptysis, and diarrhea [3]. Because of the sudden nature of the outbreak and the infectious power of the virus, it will inevitably cause people anxiety and other stress reactions, especially those diagnosed with hospitalization [4]. Besides, patients need to be isolated from their families when they are hospitalized, which increases anxiety and insomnia[5]. These emotional problems may reduce immunity and compromise recovery. 
As we have known, mind-body exercises such as Chinese traditional qigong could reduce levels of anxiety and depression in patients $[6,7]$. Baduanjin is one of the most widely practiced forms of Chinese traditional qigong and dates back nearly 2500 years [8]. It is a low-weight-bearing exercise and it is a mind/body exercise that focuses on promoting the circulation of vital energy to regulate the body, mind, and breathing. Previous studies showed that Baduanjin exercise was effective for people in anxiety and insomnia [9-11]. A Systematic Review indicated the efficacy of Baduanjin exercise in reducing anxiety symptoms in people with physical or mental illnesses [9]. However, scientific evidence has been lacking as to whether the Baduanjin exercise in COVID-2019 people may also have the potential to improve the anxiety and insomnia.

The present study was designed to use the Generalized Anxiety Disorder (GAD-7)[12] and St. Mary's Hospital Sleep Questionnaire (SMH Sleep Questionnaire) [13]to evaluate the possible beneficial effects of Baduanjin exercise in improving the anxiety and insomnia in COVID-2019 patient who practiced Baduanjin as their major daily physical activity during hospitalization.

\section{Methods}

\section{Participants}

The COVID-2019 patients who were admitted to Hubei Provincial Hospital of Integrated Chinese\& Western Medicine and who accepted to doing Baduanjin exercise were included in the study. Exclusion criteria included: cardiogenic shock, severe heart failure (cardiac function IV according to the NYHA), active bleeding, or muscle strength was lower than grade 4 . A total of 130 patients responded and completed a questionnaire about their medical history, lifestyle, and mental status. 39 patients met the inclusion criteria because they had done Baduanjin before or willing to do Baduanjin. During the hospitalization, the patients included in the study were asked to continue making Baduanjin exercise 2 times a day and we also had professional staff to provide guidance. Baduanjin exercise consists of sitting and standing forms as shown in Supplementary Appendix Fig. 1(ab), and we also made a video as shown in Supplementary Appendix Video 1. Sitting Baduanjin suits the patient on the bed to exercise, and standing Baduanjin suits the patient who gets off the bed. Using these same exclusion criteria, 39 COVID-2019 patients were recruited as controls. These patients were engaged in only minor irregular physical exercise. Data about gender, age, Hypertension, WBC, NEUR, Time of onset to admission, Ribavirin Injection, Moxifloxacin sodium chloride injection, Oseltamivir, etc, of patients in the two groups, are shown in Table 1. All patients prospectively completed the GAD-7 Questionnaire and SMH Sleep Questionnaire as part of standard clinical care. Finally, we compared the GAD-7 scores and the SMH Sleep Questionnaire scores of patients on baseline and discharge. The study protocol was approved by the Ethics Committee of Guangdong Provincial Hospital of Chinese Medicine (Ethical approval number, ZE2020-032-01) and all participants read and signed an informed consent form. 
Table 1

Participant's demographic characteristics for both groups: Baduanjin exercise and control

\begin{tabular}{|c|c|c|c|}
\hline Variable & $\begin{array}{l}\text { Baduanjin Group }(n= \\
\text { 39) }\end{array}$ & $\begin{array}{l}\text { Control Group }(\mathrm{n}= \\
39)\end{array}$ & $P$ \\
\hline Age,years & $57.23 \pm 14.37$ & $60.54 \pm 16.34$ & 0.306 \\
\hline Male,n (\%) & $17(43.6 \%)$ & $16(41.0 \%)$ & 0.819 \\
\hline Time of onset to admission & $13.49 \pm 10.09$ & $11.28 \pm 9.24$ & 0.318 \\
\hline Ribavirin Injection n(\%) & $35(89.7 \%)$ & $34(87.2)$ & 0.723 \\
\hline $\begin{array}{l}\text { Moxifloxacin sodium chloride injection } n \\
(\%)\end{array}$ & $37(94.9 \%)$ & $38(97.4 \%)$ & 0.552 \\
\hline Oseltamivir $\mathrm{n}(\%)$ & $30(76.9 \%)$ & $29(74.4 \%)$ & 0.792 \\
\hline Methylprednisolone n(\%) & $36(92.3 \%)$ & $35(89.7 \%)$ & 0.692 \\
\hline Hypertension, n (\%) & $4(10.3 \%)$ & $5(12.8 \%)$ & 0.723 \\
\hline WBC(G/L) & $5.87 \pm 3.21$ & $6.01 \pm 2.93$ & 0.851 \\
\hline Lymphocyte percentage(\%) & $27.32 \pm 12.04$ & $23.80 \pm 17.76$ & 0.322 \\
\hline NEUR(\%) & $65.29 \pm 14.89$ & $67.44 \pm 17.94$ & 0.566 \\
\hline $\mathrm{CRP}(\mathrm{mg} / \mathrm{L})$ & $32.71 \pm 45.01$ & $35.93 \pm 57.37$ & 0.285 \\
\hline CK (U/L) & $75.55 \pm 62.02$ & $97.07 \pm 99.07$ & 0.268 \\
\hline CK-MB(U/L) & $8.60 \pm 6.97$ & $11.63 \pm 23.30$ & 0.420 \\
\hline D dimer (mg/L) & $3.01 \pm 7.80$ & $4.26 \pm 10.50$ & 0.620 \\
\hline Calcitonin (ng/L) & $0.06 \pm 0.09$ & $0.11 \pm 0.15$ & 0.074 \\
\hline Troponin(ng/ml) & $0.003 \pm 0.005$ & $0.036 \pm 0.186$ & 0.277 \\
\hline creatinine $(\mu \mathrm{mol} / \mathrm{L})$ & $66.92 \pm 15.77$ & $70.74 \pm 18.40$ & 0.329 \\
\hline
\end{tabular}

\section{Outcome measures}

\section{The GAD-7 measurement}

The GAD-7 is a valid and efficient tool for screening for GAD and assessing its severity in clinical practice and research. It is efficient in that it is brief and can be completed entirely by the patient. The internal consistency of the GAD-7 was excellent (Cronbach $=0.92$ ). Test-retest reliability was also good (intraclass correlation $=0.83$ ).

The GAD-7 is a seven-item screening measure of anxiety. Each Item has five possible answer options ranging from "not at all," "several days," "more than half the days," and "nearly every day," scored as 0,1 , 
2, and 3, respectively. The GAD-7 scale score ranges from 0 to 21 [12].

\section{SMH Sleep Questionnaire}

The St. Mary's Hospital (SMH) Sleep Questionnaire was designed to evaluate the sleep of hospital patients [13]. We used a modified Chinese version which consists of 7 questions evaluating sleep and early-morning behavior over the previous $24 \mathrm{~h} \mathrm{[14].} \mathrm{The} \mathrm{internal} \mathrm{consistency} \mathrm{of} \mathrm{the} \mathrm{SMH} \mathrm{Sleep}$ Questionnaire was excellent (Cronbach $=0.72-0.85$ ). The questionnaire is a self-assessment scale developed to evaluate the sleep problems of hospitalized patients. It includes sleep quality, sleep satisfaction, daytime function, dysfunction, and frequency of night waking. The scale is characterized by a small number of questions, simplicity, and high repeatability. If the score on the scale is higher, the better the patient's sleep.

\section{Statistics}

Student T-test and Chi-Square tests were used to verify possible differences between groups in terms of socio-demographic data. Paired $t$-tests were used to compare the changes in each group between baseline and discharge measurements. Independent 2-tailed Student t-tests were used to detect differences in the GAD-7 and the SMH Sleep Questionnaire between the 2 groups at baseline and discharge. The statistical significance level was set at P less than 0.05 . The SPSS statistical program, version 18.0, for Windows was used for the tests.

\section{Results}

\section{Comparison of Subject Distribution}

Table 1 shows the homogeneity in age, gender, Time of onset to admission, Ribavirin Injection, Moxifloxacin sodium chloride injection, Oseltamivir, Methylprednisolone, Hypertension, WBC, NEUR, etc between the Baduanjin Group and Control Groups.

\section{Comparison of GAD-7 score between baseline and discharge}

Table 2 summarizes the differences between the two groups at baseline and discharge. The paired T-test showed that two groups had improved the GAD-7 scores compared with baseline at discharge. Also, independent Student t-tests found that the two groups of COVID-19 patients had no difference in the GAD-7 score at baseline. However, it revealed that the GAD-7 score in the Baduanjin group was $43.9 \%$ lower $(p<0.001)$ than in the control group at discharged. 
Table 2

Differences in the GAD-7 score between Baduanjin exerciser and Control

\begin{tabular}{|c|c|c|c|c|c|}
\hline & & $\begin{array}{l}\text { Baduanjin Group }(n= \\
\text { 39) }\end{array}$ & $\begin{array}{l}\text { Control Group }(\mathrm{n}= \\
39)\end{array}$ & $\begin{array}{l}\text { Difference } \\
(\%)\end{array}$ & $P$ \\
\hline Baseline & $\begin{array}{l}\text { GAD-7 } \\
\text { score }\end{array}$ & $13.12 \pm 2.94$ & $12.36 \pm 4.98$ & 61.6 & 0.409 \\
\hline \multirow[t]{3}{*}{ Discharge } & $\begin{array}{l}\text { GAD-7 } \\
\text { score }\end{array}$ & $0.36 \pm 0.63$ & $2.53 \pm 2.25$ & 43.9 & $\begin{array}{l}< \\
0.001 *\end{array}$ \\
\hline & $\mathrm{T}$ & 26.47 & 11.862 & & \\
\hline & $P$ & $<0.001^{\#}$ & $<0.001^{\#}$ & & \\
\hline
\end{tabular}

NOTE. Data are mean \pm SD.

* $\mathrm{P}<0.001$ (independent Student $\mathrm{t}$ test); ${ }^{\#} \mathrm{P}<0.001$ (Paired $\mathrm{t}$ test)

\section{Comparison of SMH Sleep Questionnaire between baseline and discharged}

Table 3 summarizes the differences between the two groups at baseline and discharge. According to statistics, it was found that the SMH Sleep Questionnaire score in each group was improved when they were discharged from the baseline. Independent Student t-tests found that the two groups had no difference in the SMH Sleep Questionnaire score at baseline. However, at discharge, the SMH Sleep Questionnaire score in the Baduanjin group was $75.9 \%$ higher $(p=0.003)$ than in the control group.

Table 3

Differences in the SMH Sleep Questionnaire between Baduanjin exerciser and Control

\begin{tabular}{|c|c|c|c|c|c|}
\hline & & $\begin{array}{l}\text { Baduanjin Group(n } \\
=39)\end{array}$ & $\begin{array}{l}\text { Control Group(n } \\
=39)\end{array}$ & $\begin{array}{l}\text { Difference } \\
(\%)\end{array}$ & $P$ \\
\hline Baseline & $\begin{array}{l}\text { SMH Sleep } \\
\text { Questionnaire }\end{array}$ & $17.90 \pm 3.30$ & $17.08 \pm 3.53$ & 76 & 0.292 \\
\hline \multirow[t]{3}{*}{ Discharge } & $\begin{array}{l}\text { SMH Sleep } \\
\text { Questionnaire }\end{array}$ & $26.08 \pm 4.10$ & $23.18 \pm 4.28$ & 75.9 & $0.003^{*}$ \\
\hline & $\mathrm{T}$ & 9.709 & 7.190 & & \\
\hline & $P$ & $<0.001^{\#}$ & $<0.001^{\#}$ & & \\
\hline \multicolumn{6}{|c|}{ NOTE. Data are mean \pm SD. } \\
\hline \multicolumn{6}{|c|}{ * $\mathrm{P}<0.05$ (independent Student $\mathrm{t}$ test); ${ }^{*} \mathrm{P}<0.001$ (Paired $\mathrm{t}$ test) } \\
\hline
\end{tabular}

\section{Discussion}


In this study we found that Baduanjin exercise presented a better performance in the GAD-7 and SMH Sleep Questionnaire when compared to the control group, probably indicating that Baduanjin exercise is beneficial to regulating COVID-19 patients' mood and promoting their sleep. This is the first study to report that Baduanjin exercise promotes anxiety and insomnia in COVID-19 patients.

The COVID-19 outbreak has reached every continent on Earth besides Antarctica. The respiratory disease, caused by a never-before-seen coronavirus, has upended life across the globe, shutting down entire cities and countries in a matter of months. The economy has come to a screeching halt. The epicenter-Wuhan, China - experienced the worst of the initial outbreak but appears to be getting things under control whereas huge, secondary outbreaks have appeared in Europe and the US. COVID-19 is classified as an airborne high consequence of infectious disease (HCID) in the world. The COVID-19 symptoms may begin like the flu but go on to develop fever, cough, and shortness of breath that is severe enough to warrant hospitalization in many patients [3]. Unfortunately, to date, there is no antiviral drug or vaccine to treat this infection. The management of patients mainly focuses on the provision of supportive care, e.g, oxygenation, ventilation, fluid management, and psychological nursing [15].

A national survey from the American Psychiatric Association (APA) shows COVID-19 is seriously affecting Americans' mental health, with half of US adults reporting high levels of anxiety [16]. Besides, a survey study shows that during the initial phase of the COVID-19 outbreak in China, more than half of the respondents rated the psychological impact as moderate-to-severe, and about one-third reported moderate-to-severe anxiety [17]. People especially the patient tend to misinterpret benign bodily sensations and changes as dangerous. This will increase their anxiety, influence their ability to make rational decisions, and impact their behavior [18]. As we have known, Baduanjin exercise is a lowintensity, aerobic exercise and several studies have demonstrated other benefits of Baduanjin exercise, including relieving pain and stiffness, and improving sleep quality and psychological well-being $[11,19$, 20]. In our study, we found the two groups had improved the GAD-7 scores and SMH Sleep Questionnaire compared with baseline, which may be related to the successful discharge of COVID-19 patients. Nevertheless, the significant differences found in the Baduanjin group included a $43.9 \%$ lower $(p<0.001)$ in the GAD-7 score and an approximately 75.9\% higher $(p=0.003)$ in SMH Sleep Questionnaire score compare with the control group. The results of this study suggest that the Baduanjin exercise might be beneficial to the physical and mental rehabilitation of COVID-19 patients. Therefore, Baduanjin exercise may be used as a rehabilitation exercise for COVID-19 patients when they are in hospital.

For many of us, the coronavirus and the COVID-19 illness make for a very uncertain future. People worry about their own health and the health of their loved ones, here and abroad. While anxiety is a normal and expected reaction to the pandemic, too much anxiety can start to cause harm. Feeling stressed and fearful every day takes a toll on health and well-being very quickly. Hence, you can use that concern to take positive and protective actions-things like practicing good hygiene, having a plan in case you need to self-isolate, and taking Baduanjin exercise to relax mood. 
This study also has some limitations. First, our study was retrospective and subject to self-selection bias. There are a lot of confounding factors that can't be controlled. Second, the number of subjects recruited for the study was relatively small. Third, the GAD-7 and SMH Sleep Questionnaire were not evaluated continuously during hospitalization, making it impossible to know exactly when the two groups differed. Nonetheless, this study provides some basis for the Baduanjin exercise to promote the recovery of COVID19 patients. In the future, more research is still needed to confirm this conclusion.

\section{Conclusions}

The Baduanjin exercise may improve the anxiety and insomnia in COVID-2019 patients. It can also be used as a form of rehabilitation exercise for discharged patients or patients isolated at home.

\section{Abbreviations}

COVID-19

the novel coronavirus 2019;

\section{Declarations}

\section{Acknowledgements}

The authors gratefully acknowledge the contributions of all the investigators, and participants of the trial. This study was financially supported by the scientific and technological research of traditional Chinese medicine, Guangdong Provincial Hospital of Chinese Medicine(No.YN2016HL03).

\section{Authors' contributions}

MGC, RXZ, XZ, MZZ, ML designed the study and contributed to thewriting of the protocol. MGC and EC were responsible for collecting the consent forms from the study participants, collecting and safeguarding all study data during the study period, and writing the first draft of the manuscript. $\mathrm{HC}$ designed and conducted the statistical analyses. QW, XZ and ML provided substantial background material, conducted literature searches and provided contextual information for the final manuscript. All authors contributed to and approved the final manuscript.

\section{Funding}

None

\section{Availability of data and materials}

The data used to support the findings of this study are available from the corresponding author upon request. 


\section{Ethics approval and consent to participate}

This study protocol was approved by the Ethics Committee of Guangdong Provincial Hospital of Chinese Medicine (Ethical approval number, ZE2020-032-01) and all participants read and signed an informed consent form.

\section{Consent for publication}

Not applicable.

\section{Competing interests}

The authors declare that they have no competing interests.

\section{Author details}

${ }^{1}$ Intensive care unit, The Second Clinical College of Guangzhou University of Chinese Medicine, Guangzhou, 510120, China. ${ }^{2}$ Nursing department, The Second Clinical College of Guangzhou University of Chinese Medicine, Guangzhou, 510120, China.

\section{References}

1. Singhal T. 'A Review of Coronavirus Disease-2019 (COVID-19)'. Indian J Pediatr. 2020;87(4):281-6.

2. Velavan TP. and C. G. Meyer: 'The COVID-19 epidemic'. Tropical Medicine International Health. 2020;25:278-80.

3. Chen ZM, Fu JF, Shu Q, Chen YH, Hua CZ, Li FB, Lin R, Tang LF, Wang TL, Wang W, Wang YS, Xu WZ, Yang ZH, Ye S, Yuan TM, Zhang CM, and Y. Y. Zhang: 'Diagnosis and treatment recommendations for pediatric respiratory infection caused by the 2019 novel coronavirus', World journal of pediatrics: WJP, 2020.

4. Wang Y, Di Y, Ye J, Wei W. 'Study on the public psychological states and its related factors during the outbreak of coronavirus disease 2019 (COVID-19) in some regions of China', Psychology, health \& medicine, 2020, 1-10.

5. Balanza-Martinez V, Atienza-Carbonell B, Kapczinski F. and R. B. De Boni: 'Lifestyle behaviours during the COVID-19 - time to connect', Acta psychiatrica Scandinavica, 2020.

6. Ren Y, Li M. 'Influence of physical exercise on social anxiety of left-behind children in rural areas in China: The mediator and moderator role of perceived social support'. J Affect Disord. 2020;266:2239.

7. Li Z, Liu S, Wang L, Smith L. 'Mind-Body Exercise for Anxiety and Depression in COPD Patients: A Systematic Review and Meta-Analysis', International journal of environmental research and public health, 2019, 17(1). 
8. Chen M, Zeng RX, Liang X, Hu X, Kong L, Wang J, Guo L, Zhang MZ, Zhang X. 'Seated-Baduanjin as an adjuvant rehabilitation treatment for dysfunctional ventilatory weaning response: A case report'. Medicine. 2018;97(34):e11854.

9. Zou L, Yeung A, Quan X, Boyden SD, Wang H. 'A Systematic Review and Meta-Analysis of Mindfulness-Based (Baduanjin) Exercise for Alleviating Musculoskeletal Pain and Improving Sleep Quality in People with Chronic Diseases', International journal of environmental research and public health, 2018, 15(2).

10. Jiang YH, Tan C, Yuan S. 'Baduanjin Exercise for Insomnia: A Systematic Review and Meta-Analysis', Behavioral sleep medicine, 2017, 1-13.

11. Chan JS, Ho RT, Chung KF, Wang CW, Yao TJ, Ng SM, and C. L. Chan: 'Qigong exercise alleviates fatigue, anxiety, and depressive symptoms, improves sleep quality, and shortens sleep latency in persons with chronic fatigue syndrome-like illness', Evidence-based complementary and alternative medicine: eCAM, 2014, 2014, 106048.

12. Spitzer RL, Kroenke K, Williams JBW, Lowe B. 'A Brief Measure for Assessing Generalized Anxiety Disorder'. Arch Intern Med. 2006;166:1092-7.

13. Leigh TJ, Bird HA, Hindmarch I, Constable PDL, and V. Wright: 'Factor Analysis of the St. Mary's hospital Sleep Questionnaire', Sleep, 1988, 11, 448-453.

14. Chen L: 'Reliability and validity and clinical practice of the chinese version fo Richards-Campbell Sleep Questionnaire', Dalian University, 2017.

15. Cunningham AC, Goh HP, Koh D. 'Treatment of COVID-19: old tricks for new challenges', Critical Care, 2020, 24(1).

16. Anon. 'American Psychiatric Association Website: www.medscape.com.'.

17. Wang C, Pan R, Wan X, Tan Y, Xu L, Ho CS, Ho RC. 'Immediate Psychological Responses and Associated Factors during the Initial Stage of the 2019 Coronavirus Disease (COVID-19) Epidemic among the General Population in China', International journal of environmental research and public health, 2020, 17(5).

18. Asmundson GJG, Taylor S. 'How health anxiety influences responses to viral outbreaks like COVID19: What all decision-makers, health authorities, and health care professionals need to know'. J Anxiety Disord. 2020;71:102211.

19. Chan JS, Li A, Ng SM, Ho RT, Xu A, Yao TJ, Wang XM, So KF. and C. L. Chan: 'Adiponectin Potentially Contributes to the Antidepressive Effects of Baduanjin Qigong Exercise in Women With Chronic Fatigue Syndrome-Like Illness'. Cell Transplant. 2017;26(3):493-501.

20. Ying W, Min QW, Lei T, Na ZX, Li L, Jing L. 'The health effects of Baduanjin exercise (a type of Qigong exercise) in breast cancer survivors: A randomized, controlled, single-blinded trial'. European journal of oncology nursing: the official journal of European Oncology Nursing Society. 2019;39:90-7.

\section{Supplementary Files}


This is a list of supplementary files associated with this preprint. Click to download.

- SupplementaryAppendixfigure1.pdf

- SupplementaryAppendixfigure1.pdf 